\title{
Screening Effects on Electron-Capture Rates and Type la Supernova Nucleosynthesis
}

\author{
Toshio Suzuki ${ }^{1,8, *}$, Kanji Mori ${ }^{2,8}$, Michio Honma ${ }^{3}$, Michael A. Famiano ${ }^{4,8}$, Toshitaka \\ Kajino $^{5,6,8}$, Motohiko Kusakabe ${ }^{5,8}$, and A. Baha Balantekin ${ }^{7,8}$ \\ ${ }^{1}$ Department of Physics, College of Humanities and Sciences, Nihon University, 3-25-40 Sakurajosui, \\ Setagaya-ku, Tokyo 156-8550, Japan \\ ${ }^{2}$ Research Institute of Stellar Explosive Phenomena, Fukuoka University, 8-19-1 Nanakuma, Jonan-ku, \\ Fukuoka-shi, Fukuoka 814-0180, Japan \\ ${ }^{3}$ Center for Mathematical Sciences, University of Aizu, Aizu-Wakamatsu, Fukushima 965-8580, Japan \\ ${ }^{4}$ Department of Physics, Western Michigan University, Kalamazoo, MI 49008, USA \\ ${ }^{5}$ School of Physics, Beihang University, Haidian-qu, Beijing 100083, People's Republic of China \\ ${ }^{6}$ Graduate School of Science, The University of Tokyo, Hongo, Bunkyo-ku, Tokyo 113-0033, Japan \\ ${ }^{7}$ Department of Physics, University of Wisconsin-Madison, Madison, WI 53706, USA \\ ${ }^{8}$ National Astronomical Observatory of Japan, 2-21-2 Osawa, Mitaka, Tokyo 181-8588, Japan
}

\begin{abstract}
Nucleosynthesis of iron-group elements in Type Ia supernovae is studied for single-degenerate models with the use of electron-capture rates updated with the new shell-model Hamiltonian in $p f$-shell. An over-production problem of neutron-rich iron-group isotopes compared with the solar abundances is now found to be suppressed within a factor of about twice for the updated weak rates. Effects of screening on nucleosynthesis are investigated for explosion models of fast deflagration and slow deflagration with delayed detonation. The e-capture rates are reduced by the screening, especially by the screening effects on the ions. The production yields of most neutron-rich isotopes such as ${ }^{50} \mathrm{Ti},{ }^{54} \mathrm{Cr}$ and ${ }^{58} \mathrm{Fe}$ are found to be suppressed most by the screening. The inclusion of the screening is desirable for precise evaluation of abundances of neutron-rich nuclides.
\end{abstract}

\section{Introduction}

First, we show that a new shell-model Hamiltonian, GXPF1J [1], can describe Gamow-Teller (GT) strengths and e-capture rates in $p f$-shell nuclei quite well. Electron-capture rates at stellar environments are evaluated by using GT strength and phase space factor for the reaction. Electron chemical potential, thus the phase space factor also, increases as the density increases and the e-capture rates are enhanced at high densities. In the pioneering work of Fuller, Fowler and Newton (FFN) [2], a simple shell model was used to evaluate the GT strength. Now, large-scale shell-model calculations are possible for $p f$-shell nuclei. Typical Hamiltonians are KB3's and GXPF1's. While both can describe spin properties of $p f$-shell nuclei very well, experimental GT strength distributions in ${ }^{58} \mathrm{Ni}$, for example, are better reproduced for GXPF1J than KB3G [3] in both $\mathrm{n} \rightarrow \mathrm{p}$ and $\mathrm{p} \rightarrow \mathrm{n}$ channels. The GXPF1J can also reproduce $B(M 1)$ in ${ }^{48} \mathrm{Ca}$ and experimental e-capture rates in $p f$-shell nuclei best among various shell-model and RPA calculations [4].

\footnotetext{
*e-mail: suzuki.toshio@nihon-u.ac.jp
} 
We here discuss ${ }^{56} \mathrm{Ni}$ in particular. The experimental GT strength obtained by $(\mathrm{p}, \mathrm{n})$ reaction has two-peak structure [5], and it can be well reproduced by GXPF1J [6] while KB3G and KBF [7] give only one peak. As the strength is more spread for GXPF1J, the e-capture rates of GXPF1J are smaller than those of $\mathrm{KB} 3 \mathrm{G}[8,9]$. The weak rates conventionally used for stellar calculations is $\mathrm{KBF}$ [7] rather than KB3G. In KBF, experimental data of energies and GT strength available are taken into account, and thus the e-capture rates of KBF come close to those of GXPF1J.

In Sect. 2,the e-capture rates evaluated with the GXPF1J are applied to study nucleosynthesis of iron-group elements in Type Ia supernovae (SN). The screening effects on the rates and nucleosynthesis are discussed in Sect. 3.

\section{Nucleosynthesis of iron-group nucleides in Type la SN}

We discuss synthesis of iron-group nucleides in Type Ia SN. Here, we treat single-degenerate 'Chandra' model, where accretion of matter to a white dwarf (WD) from non-degenerate companion occurs. A SN explosion ( $\mathrm{SNe}$ ) occurs when WD mass approaches the Chandrasekhar limit, and much ${ }^{56} \mathrm{Ni}$ is produced in the explosion. Successive e-capture processes produce neutron-rich (n-rich) nuclei and the electron-fraction $Y_{e}$ decreases. Decrease of ecapture rates leads to less production of n-rich nuclei and larger $Y_{e}$.

There was an over-production problem of neutron-excess iron-group isotopes compared with the solar abundances when e-capture rates of FFN were used. Neutron-rich nuclei such as ${ }^{54} \mathrm{Cr}$ and ${ }^{58} \mathrm{Ni}$ are produced several times more than the solar abundances when an explosion model W7 with fast deflagration with subsonic frame front propagation was used [10]. Another explosion model WDD2 [10] with slow deflagration and delayed detonation with supersonic shock wave will be used also. The production yields of elements are obtained with the e-capture rates of GXPF1J $(21 \leq Z \leq 32)$ and KBF (other $Z$ ) for both the W7 and WDD2 explosion models [9]. The over-production of n-rich iron-group nuclides found for the FFN rates is now suppressed within a factor of about twice, and this improvement proves to be better for WDD2 [9] (see figures 1 and 2 in Sect. 3).

\section{Screening effects on the weak rates and nucleosynthesis}

\subsection{Screening effects on the weak rates}

There are two kinds of screening (SCR) effects. One is SCR effects of electrons, which is evaluated for relativistic degenerate electron liquid with the dielectric function obtained by RPA [11]. The ion-electron attraction is reduced by SCR, which leads to the reduction of ecapture rates and the enhancement of the $\beta$-decay rates. The other is the change of threshold energy due to the change of the chemical potential of the ion.

The correction of the chemical potential of the ion with charge $Z$ is given by $\mu_{C}(Z)=$ $k T f(\Gamma)$, where $\Gamma=Z^{5 / 3}\left(\frac{e^{2}}{k T a_{e}}\right)$ with $a_{e}=\left(\frac{3}{4 \pi n_{e}}\right)^{1 / 3}$ and $n_{e}$ the electron density is the Coulomb coupling parameter. For the strong-coupling regime $(\Gamma>1)$, results of Monte-Carlo calculations for one-component plasma [12] have been parametrized by a function of $\Gamma$ [13]. For the weak-coupling regime $(\Gamma<0.1), f(\Gamma)$ is obtained by cluster-expansion method [14].

$$
\begin{aligned}
& f(\Gamma)=-\frac{\Gamma^{3 / 2}}{\sqrt{3}}-\frac{\Gamma^{3}}{2}\left[\frac{3}{4} \ln (3 \Gamma)+0.57721-\frac{11}{12}\right] \quad(\Gamma<0.1) \\
& f(\Gamma)=-0.8980 \Gamma+3.8714 \Gamma^{1 / 4}-0.8828 \Gamma^{-1 / 4}-0.8610 \ln \Gamma-2.5269 \quad(\Gamma>1)
\end{aligned}
$$

The first term for the weak-coupling regime is the Debye-Huckel limit, and the second term is the next-order correction. For the intermediate coupling $(0.1<\Gamma<1)$, interpolation of 
weak and strong couplings is usually taken, but this leads to a rather complex formula [13]. Instead, a simple parametrization proposed in Ref. [15], $f(\Gamma)=-\Gamma^{3 / 2} / \sqrt{3}+\beta / \gamma \Gamma^{\gamma}$, is used here for $0<\Gamma<1$. For small $\Gamma$, it becomes the Debye-Huckel limit, and smoothly connected to the strong-coupling region at $\Gamma=1$ with $\beta=0.2956$ and $\gamma=-1.9885$. The correction of the ion chemical potential is negative and its magnitude increases as $Z$ increases. This results in an enhancement of the Q-value, $\Delta Q=\mu_{C}(Z-1)-\mu_{C}(Z)$, which leads to the reduction (enhancement) of the e-capture ( $\beta$-decay) rates [16]. Here, the SCR effects on thermonuclear reactions are also taken into account [17].

\subsection{Screening effects on the synthesis of iron-group nucleides}

Screening effects on the production yields of elements in Type Ia SNe compared with the solar abundances are investigated for the W7 and WDD2 explosion models [16]. Calculated results of the production yields obtained for the W7 and WDD2 models are shown in figure 1 and figure 2 , respectively.
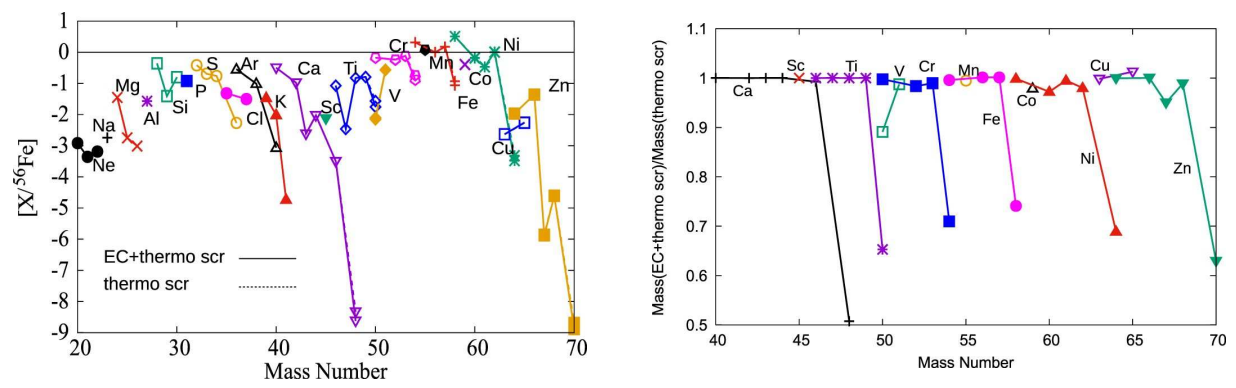

Figure 1. (Left) Abundances normalized by the solar and ${ }^{56} \mathrm{Fe}$ abundances obtained for the W7 model of SNe Ia. Solid and broken lines show results with and without the SCR effects, respectively. (Right) Abundance ratios between the cases with and without the SCR effects on e-capture rates obtained for the W7 model. Taken from [16].
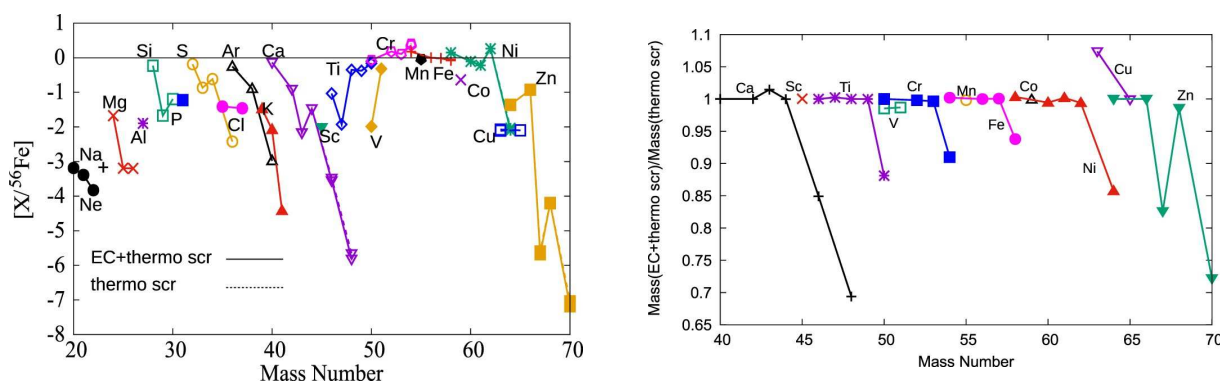

Figure 2. The same as in figure 1 for the WDD2 model of SNe Ia. Taken from [16].

The SCR effects are found to be large for the most n-rich isotopes such as ${ }^{48} \mathrm{Ca},{ }^{50} \mathrm{Ti}$, ${ }^{54} \mathrm{Cr},{ }^{58} \mathrm{Fe},{ }^{64} \mathrm{Ni}$ and ${ }^{70} \mathrm{Zn}$, and the reduction effects are smaller for WDD2 compared with W7. Most n-rich isotopes are produced more for WDD2. The yields of ${ }^{54} \mathrm{Cr}$ and ${ }^{58} \mathrm{Fe}$ are close to the solar abundances in case of WDD2, while they are one-order magnitude smaller in the W7 model. These features can be understood from the difference in $Y_{e}$, central density 
$\rho_{c}$ and central temperature $T_{c}$ between the two models as shown in figure 3. $Y_{e}$ is smaller for WDD2, which results in the production of more n-rich isotopes for the case of WDD2. $\rho_{c}$ is higher for WDD2, which leads to less effects of SCR for WDD2.
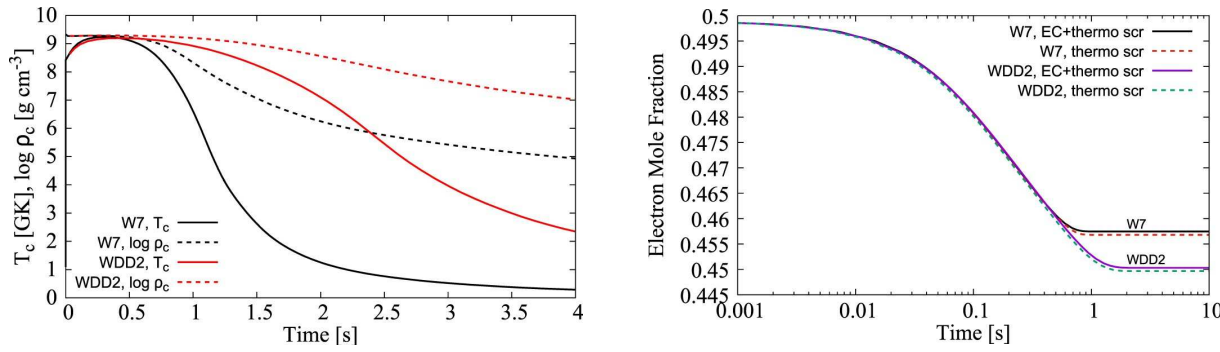

Figure 3. (Left) Time evolution of the central density $\rho_{c}$ and temperature $T_{c}$. The solid lines show $T_{c}$ and the broken lines show $\rho_{c}$. The upper (lower) lines are obtained by the WDD2 (W7) model of SNe Ia. (Right) Time evolution of the electron fraction $Y_{e}$ at the center of the SN Ia models. The solid lines show the result with the SCR effects on both e-capture and thermonuclear reactions, while the broken lines show the result with the SCR effect only on thermonuclear reactions. Taken from [16].

Finally, we discuss the contribution of SNe Ia to the solar abundances of very n-rich nuclei such as ${ }^{50} \mathrm{Ti},{ }^{54} \mathrm{Cr}$ and ${ }^{58} \mathrm{Fe}$, which are produced also in core-collapse SN. The contributions are 5-10\% in W7 and 50-100\% in WDD2. The suppressions due to the SCR effects are $30-50 \%$ in W7 and they are as small as $10-20 \%$ in WDD2. Besides the explosion models, SCR effects are important for precise evaluation of synthesis of very n-rich isotopes, ${ }^{54} \mathrm{Cr}$ and ${ }^{58} \mathrm{Fe} .{ }^{54} \mathrm{Cr}$ is still over-produced in WDD2 model. Here, use is made of post-process calculations in 1D explosion models, where hydrodynamics and nucleosynthesis are decoupled. Improvement of this defect is left to future.

\section{References}

[1] M. Honma, T. Otsuka, T. Mizusaki et al., J. Phys. Conf. Ser. 20, 7 (2005)

[2] G. M. Fuller, W. Fowler and M. J. Newman, Astrophys. J. 252, 715 (1982); Astrophys. J. Suppl. 48, 279 (1982)

[3] A. Poves, J. Sanchez-Solano, E. Caurier and F. Nowacki, Nucl. Phys. A694, 157 (2001)

[4] A. L. Cole, T. S. Anderson, R. G. T. Zegers et al., Phys. Rev. C 86, 015809 (2012)

[5] M. Sasano, G. Perdikakis, R. G. T. Zegers et al., Phys. Rev. Lett. 107, 202501 (2011)

[6] T. Suzuki, M. Honma, K. Higashiyama et al., Phys. Rev. C 79, 061603(R) (2009)

[7] K. Langanke and G. Martinez-Pinedo, Atomic Data and Nucl. Data Tables 79, 1 (2001)

[8] T. Suzuki, M. Honma, H. Mao et al., Phys. Rev. C 83, 044619 (2011)

[9] K. Mori, M. A. Famiano, T. Kajino, T. Suzuki et al.,Astrophys. J. 833, 179 (2016)

[10] K. Iwamoto, F. Brachwitz, K. Nomoto et al., Astrophys. J. Suppl. 125, 439 (1999)

[11] N. Itoh, N. Tomizawa, M. Tamamura and S. Wanajo, Astrophys. J. 579, 380 (2002)

[12] W, L. Slattery, G. D. Doolen and H. E. Dewitt, Phys. Rev. A 26, 2255 (1982)

[13] S. Ichimaru, Rev. Mod. Phys. 65, 255 (1993)

[14] R. Abe, Prog. Theor. Phys. 21, 475 (1959)

[15] D. G. Yakovlev and D. A. Shalybkov, Astrophy. Space Phys. 7, 311 (1989)

[16] K. Mori, T. Suzuki, M. Honma et al., Astrophy. J. 904, 29 (2020)

[17] R. K. Wallace, S. E. Woosley and T. A. Weaver, Astrophys. J. 258, 696 (1982) 